\title{
GPPS-BJ-2019-76
}

\section{INVESTIGATION ON THE FLAME FRONT AND FLOW FIELD IN ACOUSTICALLY EXCITED SWIRLING FLAMES WITH AND WITHOUT CONFINEMENT}

\author{
Guoqing Wang \\ Shanghai Jiao Tong University \\ wgqing@sjtu.edu.cn \\ Shanghai, China
}

\author{
Xunchen Liu \\ Shanghai Jiao Tong University \\ liuxunchen@sjtu.edu.cn \\ Shanghai, China
}

\author{
Lei $\mathrm{Li}^{*}$ \\ Shanghai Jiao Tong \\ University \\ lilei81@sjtu.edu.cn \\ Shanghai, China
}

\author{
Zhi X. Chen \\ University of Cambridge \\ zc252@cam.ac.uk \\ Cambridge, United Kingdom
}

\author{
Fei Qi \\ Shanghai Jiao Tong \\ University \\ fqi@sjtu.edu.cn \\ Shanghai, China
}

\begin{abstract}
Open and confined dimethyl ether/air swirling flames were investigated with an acoustically excited swirling burner. Simultaneous particle-image velocimetry and formaldehyde planar laser-induced fluorescence were measured using a high repetition rate burst-mode laser at 20 $\mathrm{kHz}$. Time-averaged velocity field, vorticity and flame brush cloud were compared under different confinements and acoustic excitation conditions. The integrated fluorescence intensities are similar while the open flame has dramatically large fluorescence areas. Time-resolved velocity field and flame brush were measured to investigate the continuous evolution of swirling flame front and acoustically induced vortex. The flame brush was found to be much dispersed in the open flame due to the mixing effect from the surrounding air. Flame root and flame angle were extracted and analyzed statistically using the fluorescence images. Different stages of acoustically induced vortex were observed in both confined and open cases. The vortex trajectory along the outer shear layer determines that the flame tip can perceive the vortex instead of the flame base. The flame regions affected and unaffected by acoustically induced vortex indicate different perturbation mechanisms for the heat release.
\end{abstract}

\section{INTRODUCTION}

More stringent emission regulations continually motivate the development of swirling combustion and progress of low emission techniques. Swirling flame is a kind of important flame in gas turbine and aero-engine combustors. Coupling of flame and acoustic wave can lead to dramatically unsteady heat release and pressure oscillation which are destructive to the combustor (Huang and Yang, 2009; Poinsot, 2017). Thermoacoustic instability is therefore a crucial problem in developing new engines. Numerous studies have dedicated to thermoacoustic instability of swirling flame to uncover the instability mechanism, flame dynamics, control method and so on (Huang and Yang, 2009).

Practical swirling flame usually locates in the annular combustor. Each flame experiences the confinement and interaction with adjacent flames and walls. The flow field and flame response were found to change significantly when the flame confinement varies (Fu et al., 2005; De Rosa et al., 2015; De Rosa et al., 2017). Before studying confinement effects on swirling flame, Birbaud et al. (Birbaud et al., 2007) investigated inverted conical flames on a bluff-body. They found that the dynamics of flame tip, flame wrinkling and amplitude of response are all greatly related to the confinement. Cuquel et al. (Cuquel et al., 2013) studied laminar premixed conical flames and suggested that the confinement and expansion ratio need to be taken into account in the description of the flame transfer function. $\mathrm{Fu}$ et al. (Fu et al., 2005) subsequently measured the flow field of swirling flow and found that the size and strength of the recirculation zone are highly affected by the level of confinement. De Rosa et al. (De Rosa et al., 2015; De Rosa et al., 2017) measured the shape and structure of the swirling flames using chemiluminescence imaging. The geometry of the flame in the base region was found to be insensitive to the confinement. This similarity in the location of flame base leads to a similarity in the response of this portion of the 
flame imposed oscillations in each confinement. On the basis of previous studies, it can be found that study on the difference between the externally excited swirling flame with and without wall is still scarce. The swirling flame without confinement (open flame) is a typical swirling flame configuration which can provide enough space for the full development of vortex and flame surface. Although the open flame is seldom applied for engines due to the lack of outer recirculation zone, it is still valuable to figure out the pure dynamics of swirling flame with and without wall confinement. This is thought to help understanding the swirling combustion under extreme conditions

In the past decades, phase-averaged and phase-locked techniques have played a great role in studying the flame dynamics of swirling flame under external excitation or selfexcitation. Meier et al. (Weigand et al., 2006; Meier et al., 2007) measured swirling methane/air diffusion flame and technically premixed gas turbine flame under self-excited thermoacoustic pulsations using phase-locked laser Doppler velocimetry (LDV), planar laser-induced fluorescence (PLIF), Raman scattering and chemiluminescence imaging. Bellow et al. (Bellows et al., 2006; Bellows et al., 2007) studied nonlinear flame dynamics of acoustically excited swirling flame using phase-locked OH PLIF imaging and line-of-sight chemiluminescence. Palies et al. (Palies et al., 2010) studied the response of external excited swirling flame with LDV and $\mathrm{OH}^{*}$ chemiluminescence. They found that axial velocity perturbations can induce a shedding of vortices to roll-up the flame and azimuthal perturbation can result in angular oscillation of the flame root.

Recently, some high speed laser diagnostic techniques have started to be applied in swirling flame study. Boxx et al. (Boxx et al., 2010) and Stöhr et al. (Stöhr et al., 2012) studied the transient phenomenon and interaction of helical precessing vortex core and turbulent swirl flames in a gas turbine model combustor using $5 \mathrm{kHz}$ particle image velocimetry (PIV) and OH-PLIF techniques. Allison et al. (Allison et al., 2015) applied $4 \mathrm{kHz}$ formaldehyde $\left(\mathrm{CH}_{2} \mathrm{O}\right)$ PLIF to study flame surface area fluctuations in a dual-swirl self-excited burner. Most recently, Fugger et al. (Fugger et al., 2018) performed simultaneously $\mathrm{CH}_{2} \mathrm{O}$ and $\mathrm{OH}$ PLIF and PIV at higher repetition rate of $10 \mathrm{kHz}$ while the measurement was applied to a bluff-body-stabilized turbulent premixed flame instead of swirling flame. Although kilohertz laser diagnostic techniques have been applied to study the self-excited partially-premixed turbulent swirl flames, the external acoustically excited premixed swirling flame has seldom been investigated with the high speed laser diagnostics.

In the present study, simultaneous PIV and PLIF of $\mathrm{CH}_{2} \mathrm{O}$ measurements were performed with a high repetition rate of $20 \mathrm{kHz}$. The measured PIV and PLIF results were used to investigate the flame dynamics in acoustically excited swirling flame with and without confinement. The high speed laser diagnostics provided us more transient information about the swirling flame which has not been well investigated in high time-resolution. Furthermore, the timeaveraged flow field and $\mathrm{CH}_{2} \mathrm{O}$ signal were also obtained since they provide more general characteristics of the flame after eliminating the chaotic turbulence. The continuously varied flame front and flow field under acoustic excitation were analyzed to understand the interaction and evolution of the flame surface and flow field. Dynamic flame root and flame front angle were also extracted using the fluorescence data to understand the dynamics of flame base

\section{EXPERIMENTAL SETUP AND METHODOLOGY}

A single-nozzle swirl burner (Wang et al., 2019) was applied to construct an axial swirling flame. Briefly, the burner consists of a driver unit, a settling chamber, a contraction ended by a constant diameter duct, a horizontal end piece and a burner head. Premixed mixture is controlled by two mass flow meters and delivered to the burner. The convergent unit can diminish the level of turbulence and generate a flat velocity profile before flowing through the swirler. An axial swirler is applied to rotate the flow which consists of eight twisted airfoil (NACA 8411) vanes. The swirl number is approximately 0.6 for the present swirling flow. A small bluff body is used to stabilize the flame during the unsteady motion of the flow. Two kinds of burner head, namely confined square head and open head, are used to investigate the difference between the swirling flame with and without confinement. The square head has inner size of $92 \times 92 \mathrm{~mm}$ and height of $124 \mathrm{~mm}$ corresponding to a confinement ratio of 0.22 . Four side quartz windows are mounted on the head to confine the flame and allow optical visualization. A loudspeaker is mounted at the bottom of the burner to produce an acoustic wave and modulate the swirling flame. Velocity perturbation in the gas flow is measured with a hot wire (Dantec MiniCTA) whose response frequency is up to $10 \mathrm{kHz}$.

Simultaneous PIV and $\mathrm{CH}_{2} \mathrm{O}-\mathrm{PLIF}$ were applied in this work to investigate time-resolved flow field and flame front as shown in Fig. 1. The present diagnostic system mainly depends on a high-speed burst-mode Nd:YAG laser (Spectral Energies, QuasiModo1000) with a repetition rate up to 100 $\mathrm{kHz}$ and two high-speed cameras (Photron SA-Z) with 20 $\mathrm{kHz}$ frame rate at full resolution of $1024 \times 1024$ pixels. A programmable timing unit (Lavision PTU-X) was used to synchronize all the diagnostics system. In each measurement, the two high speed cameras were simultaneously triggered to record the transmitted laser signals for about $10 \mathrm{~ms}$. The 10 ms record was mainly limited by the output time of each burst laser pulse train.

In order to measure the flow velocity, we seeded alumina particles (diameter $\sim 5 \mu \mathrm{m}$ ) in the flow and measured the Mie scattering using $532 \mathrm{~nm}$ laser beam at $20 \mathrm{kHz}$. The PIV camera recorded the Mie scattering images with a Nikkor lens (Nikkor $50 \mathrm{~mm} \mathrm{f} / 1.4 \mathrm{G}$ ) and a short band pass filter (Edmund Optics 532/10 nm). The time between laser pulses ensures that the particles can move roughly one quarter width of the interrogation window. The raw PIV image resolution is about $0.084 \mathrm{~mm} /$ pixel. Recorded PIV images were subsequently processed by the Davis 8.0 software package with a multi-pass cross correlation algorithm. The calculated velocity field resolution is about 
$0.335 \mathrm{~mm} /$ point. To capture swirling flame front dynamics, formaldehyde is selected as the maker, which has been shown to be abundant in the preheat zone of the premixed flame (Allison et al., 2015; Wabel et al., 2017). The $4_{0}^{1}$ transition in formaldehyde is excited using a frequencytripled laser at $355 \mathrm{~nm}$. The laser energy was approximately $180 \mathrm{~mJ} /$ pulse at $20 \mathrm{kHz}$. Fluorescence of $\mathrm{CH}_{2} \mathrm{O}$ was recorded by the PLIF camera and a high speed intensifier (Lambert HiCATT) which was mounted by a UV lens (Sodern Cerco $45.5 \mathrm{~mm} \mathrm{f} / 1.8$ ) and a 376-478 $\mathrm{nm}$ hendeca-band band pass filter (Semrock FF01- $\mathrm{CH}_{2} \mathrm{O}$ ). The intensifier gate time was set at $150 \mathrm{~ns}$ to eliminate chemiluminescence impact. Spatial resolution of PLIF image is about $0.084 \mathrm{~mm} / \mathrm{pixel}$.

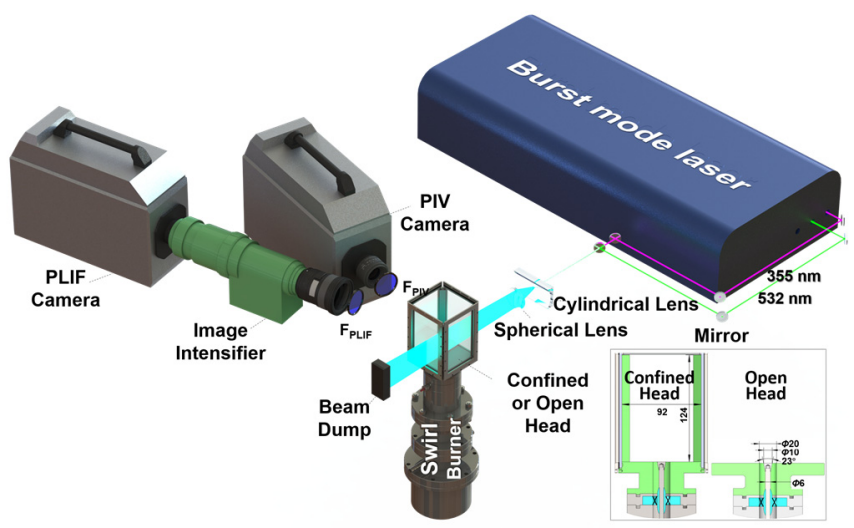

Figure 1 Schematic view of the swirl burner and the laser diagnostic system

An example of the recorded $\mathrm{CH}_{2} \mathrm{O}$ fluorescence image and the processing procedure is shown in Figure 2. After subtracting the background, the raw images are binarized with an automatically selected threshold using Otsu's method. Only the components whose area are larger than 50 pixel $^{2}\left(\sim 0.35 \mathrm{~mm}^{2}\right)$ are reserved since the typical flame components areas are larger than this value. Dilation operation is performed using a disk-shaped structuring element with a radius of 5 pixels to get the dilated region. The dilated region can cover the most possible signal of the fluorescence. The intersection of the raw image and the dilated image is then calculated applying the "and" operation. Finally, the intersection image is eroded and median filtered to get smooth edges. All the fluorescence images were normalized to the respective maximum pixel count in each time series. Using this PLIF image processing procedure, we can efficiently eliminate noise points, accurately capture $\mathrm{CH}_{2} \mathrm{O}$ fluorescence signal and retain integrity of flame front.

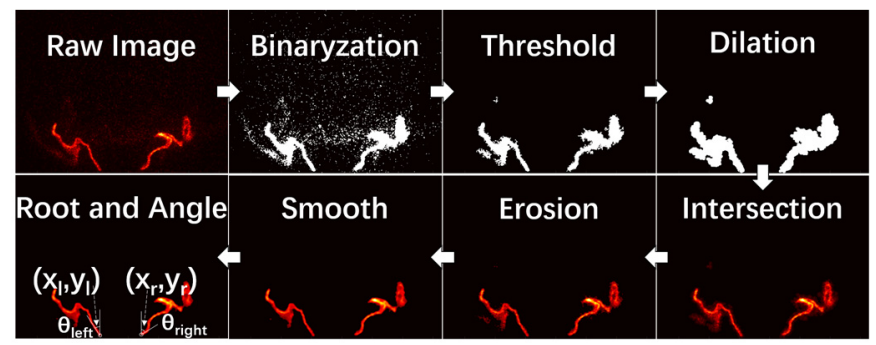

Figure 2 Illustration of image processing of $\mathrm{CH}_{2} \mathrm{O}$ fluorescence images
Experiments of dimethyl ether (DME, $\geq 99.9 \%$, Airliquide Shanghai) were performed at room temperature and atmospheric pressure using the swirling burner and laser diagnostics. DME is selected as the target fuel because it is a promising alternative fuel which can also produce significant formaldehyde concentrations in combustion. This characteristic of DME is useful in high-speed PLIF because low pulse energies, typical of high-speed laser systems, make the measurement difficult in other fuels (Allison et al., 2015). The equivalence ratio of DME/air mixtures was set at 1.0. The total flow rate was maintained at 50 SLM which corresponds to the Reynolds number of 4700 .

\section{RESULTS AND DISCUSSION}

\subsection{Averaged Flow Field}

Time-averaged flow field is investigated firstly. Figure 3 shows the averaged velocity vector and vorticity under different acoustic perturbation amplitudes. Both the flow velocity and vorticity are almost axisymmetric. Large vorticity values mainly locate near the lip of the injector at radii slightly larger than $10 \mathrm{~mm}$. The outer shear layer shows stronger vorticity than the inner shear layer. This implies that the outer shear layer is the main source of the acoustically induced vortex which can strongly influence the swirling flame front (Lieuwen, 2014). The velocity vectors are slightly affected by the acoustic modulation amplitude from 0.2 to 0.8 . However, the vorticity shows considerable variation under different modulations in Fig. 3. As the acoustic perturbation becomes stronger, the averaged vorticity values in the outer shear layer decrease while the inner shear layer becomes slightly enhanced. In general, the averaged velocity vector looks similar between the flame with and without confinement. The wall shows a weak influence on the flow field which is possibly related to the small confinement ratio of the square head.

\subsection{Averaged Fluorescence Images}

Time-averaged $\mathrm{CH}_{2} \mathrm{O}$ fluorescence images are compared in Fig. 4 for the swirling flames with and without confinement. Two distinct parts of the flame brush, which are termed left part and right part, can be observed. The two parts are not well axisymmetric since the averaged flame brush is taken from three cycles of acoustic modulation. However, some statistic characteristics of the flame, like the flame front distribution, flame angle and flame root, can still be observed from the time-averaged flame brush cloud images. The flame brush clouds distribute over larger areas compared to the instantaneous flame brush in Fig. 2. The averaged flame brush of the open flame is much dispersed than the confined flame. The flame brush cloud at $100 \mathrm{~Hz}$ and $u^{\prime} / \bar{u}=0.8$ shows that the vortex roll-up flame front nearly reaches the burner surface. Apparent fluorescence signals can be also found at height above $30 \mathrm{~mm}$ in the open flame. 


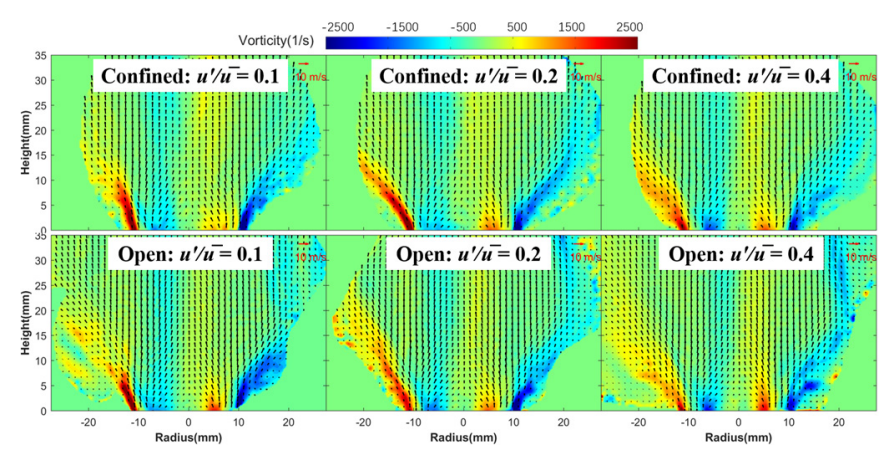

Figure 3 Averaged flow field and vorticity of DME/air swirling flame with and without confinement at $f=100 \mathrm{~Hz}$ and $u^{\prime} / \bar{u}$ from 0.2 to 0.8 .

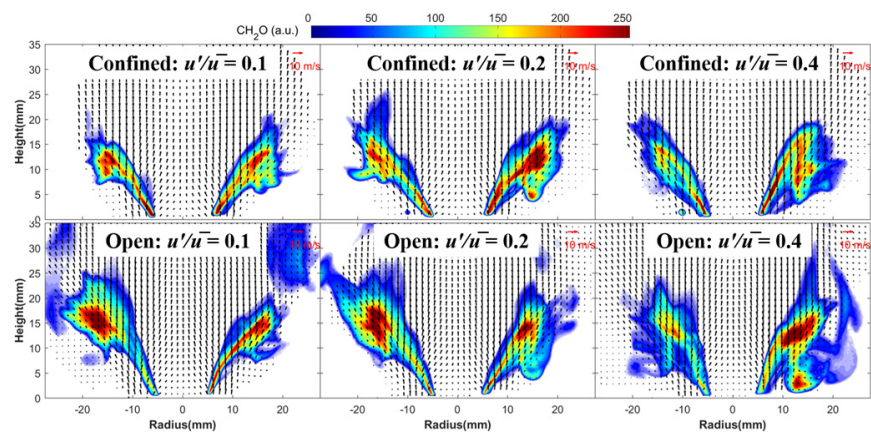

Figure 4 Averaged flow field and $\mathrm{CH}_{2} \mathrm{O}$ intensity of DME/air swirling flame with and without confinement at $f=100 \mathrm{~Hz}$ and $u^{\prime} / \bar{u}$ from 0.2 to 0.8

Although the flow fields are similar for the open and confined flames, the flame brushes show that the preheat zones are quite different. We extracted the integrated intensity and area of $\mathrm{CH}_{2} \mathrm{O}$ fluorescence signal from the images. As shown in Fig. 5, the integrated $\mathrm{CH}_{2} \mathrm{O}$ intensity in the open flame is slightly larger than that in the confined flame. Furthermore, the area of $\mathrm{CH}_{2} \mathrm{O}$ signal in the open flame is significantly larger than that in the confined flame. In other words, the DME produces more $\mathrm{CH}_{2} \mathrm{O}$ in the open flame while the mean fluorescence intensity is smaller. The dispersed $\mathrm{CH}_{2} \mathrm{O}$ signal in the peripheral region of the swirling flame contributes a lot to the large area of $\mathrm{CH}_{2} \mathrm{O}$ in the open case. The open head provides adequate space for the development of the flame front while the quartz walls of the square head only provide limited space. The flame front can therefore fully roll-up and interact with the surrounding gas which dilutes the carbon contained reactants and spreads them to far field. The confinement difference finally leads to the difference between the compacted flame front cloud of the confined swirling flames and dispersed flame front cloud of the open swirling flames. Moreover, the averaged flame brush also varies by changing the acoustic modulation. Large acoustic modulation amplitude makes the flame brush dramatically perturbed and dispersed. The larger area of the flame brush cloud is therefore observed in Fig. 4 and 5

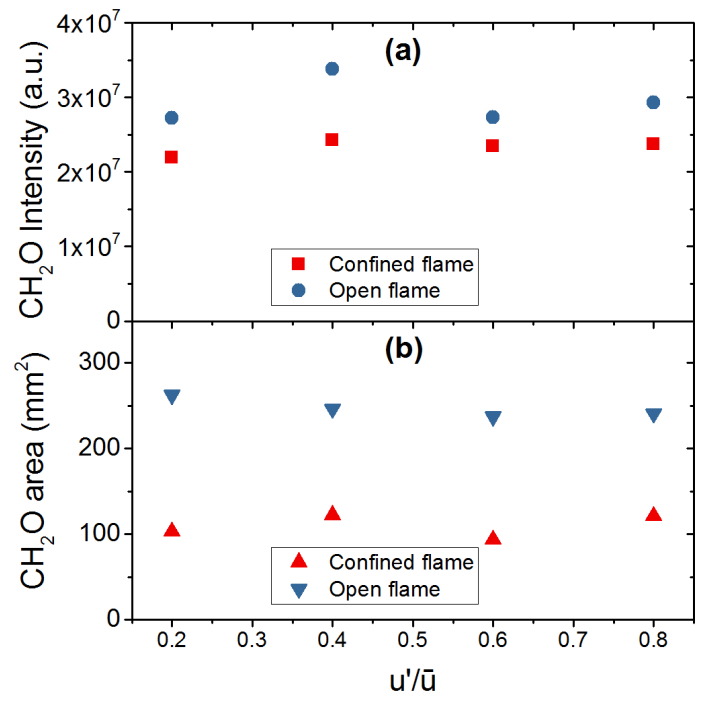

. Figure 5. The integrated intensity and area of $\mathrm{CH}_{2} \mathrm{O}$ fluorescence signal of DME/air swirling flame with and without confinement at $f=100 \mathrm{~Hz}$ and $u^{\prime} / \bar{u}$ from 0.2 to 0.8 .

\subsection{Time-Resolved Flow Field and Flame Front}

Using the high speed measurement, time-resolved flow field and $\mathrm{CH}_{2} \mathrm{O}$ PLIF were also investigated for the acoustically excited swirling flame. As shown in Figs. 6 and 7, the overlapped $\mathrm{CH}_{2} \mathrm{O}$ brush and streamlines from 0 to 9.67 ms depict the continuous evolution of the swirling flame in one acoustic cycle. The typical refraction effect can be observed from the streamlines through the flame front. This is primarily caused by the great temperature increase in the flame front (Lieuwen, 2014). The majority of the burnt gas in the central zone of the swirling flame continually flows downstream. The streamlines are more concentrated along the burner axis. Only small inner recirculation zones can be observed in the confined and open flames from $4.67 \mathrm{~ms}$ to $8.33 \mathrm{~ms}$. The weak inner recirculation zone is likely caused by the small swirl number. Moreover, the outer zone shows different characteristics from the inner zone. Distinct vortex can be observed from the peripheral zone of the swirling flame in both confined and open cases. The observed vortex is termed as acoustically induce vortex because it results from the acoustic perturbation on the flow. From the streamlines, we can observe the generation, evolution, and attenuation stages of the vortex. The vortex almost simultaneously generates from the lip of the injector and appears in pairs. It moves to the peripheral zone downstream of the flame front. The vortex size becomes larger in the movement. When the vortex arrives at the place far from the inlet mixture and flame front, the vortex loses the carried kinetic energy and gradually attenuate. A new acoustically perturbed cycle starts and produces new vortex.

Besides the flow field characteristics, the images also show the dynamically evolved flame front marked by $\mathrm{CH}_{2} \mathrm{O}$ fluorescence. The flame fronts are wrinkled and asymmetric due to the local turbulent structure. It can be recognized that the first flame brush at $0.00 \mathrm{~ms}$ and the final flame brush at $9.67 \mathrm{~ms}$ look so similar in both open and confined swirling 
flames. This provides evidence for the good repeatability between different cycles of the present acoustically excited swirling flame. In all the images, the flame base concentrates in a narrow region despite the confinement effect. They always anchor on the flow separation point of the bluff body and dynamically sway flame brush. However, the flame brush tips are quite different between the confined and open flames. The open flames show much dispersed flame front with lower intensity of the outside fluorescence signal while the confined flames have more compact flame front. The different flame front morphology implies the combustion process are changed by removing the confinement wall. The possible reason is that the surrounding air mixes with the DME and participates in the combustion reaction. The horizontal streamlines provide the evidence for the air transport. Due to the dilution and reaction effects of additional air, the swirling flame presents additional burning zone with weak flame front and low fluorescence intensity. The larger $\mathrm{CH}_{2} \mathrm{O}$ area consistent with the results in Fig. 5. The wider $\mathrm{CH}_{2} \mathrm{O}$ brush in the open case also implies that the vortex in the open case has enough space to fully develop. The fully developed vortex can further roll-up more surrounding air.

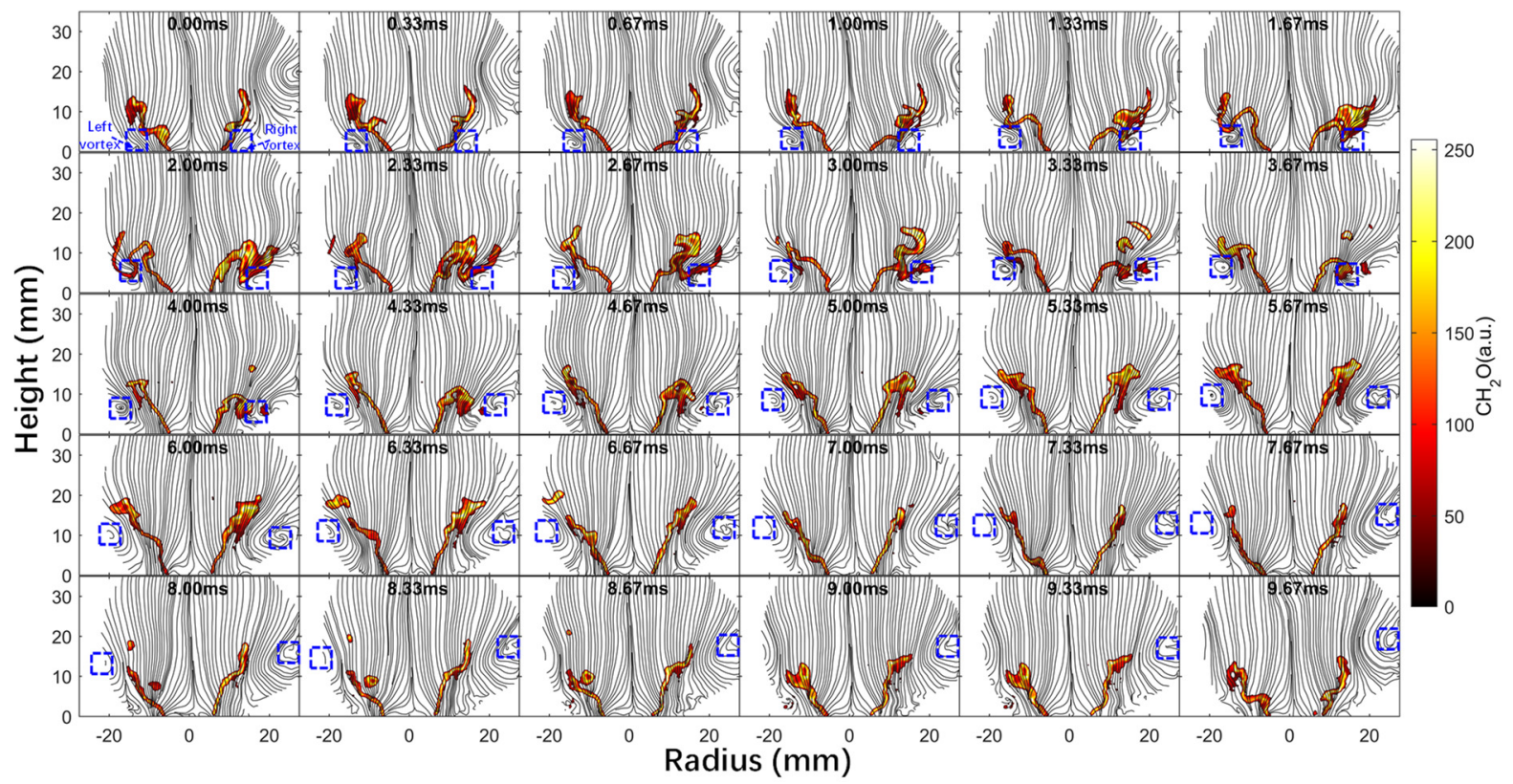

Figure 6 Time-resolved streamline and $\mathrm{CH}_{2} \mathrm{O}$ of confined DME/air swirling flame at $f=100 \mathrm{~Hz}$ and $u^{\prime} / \bar{u}=0.8$.

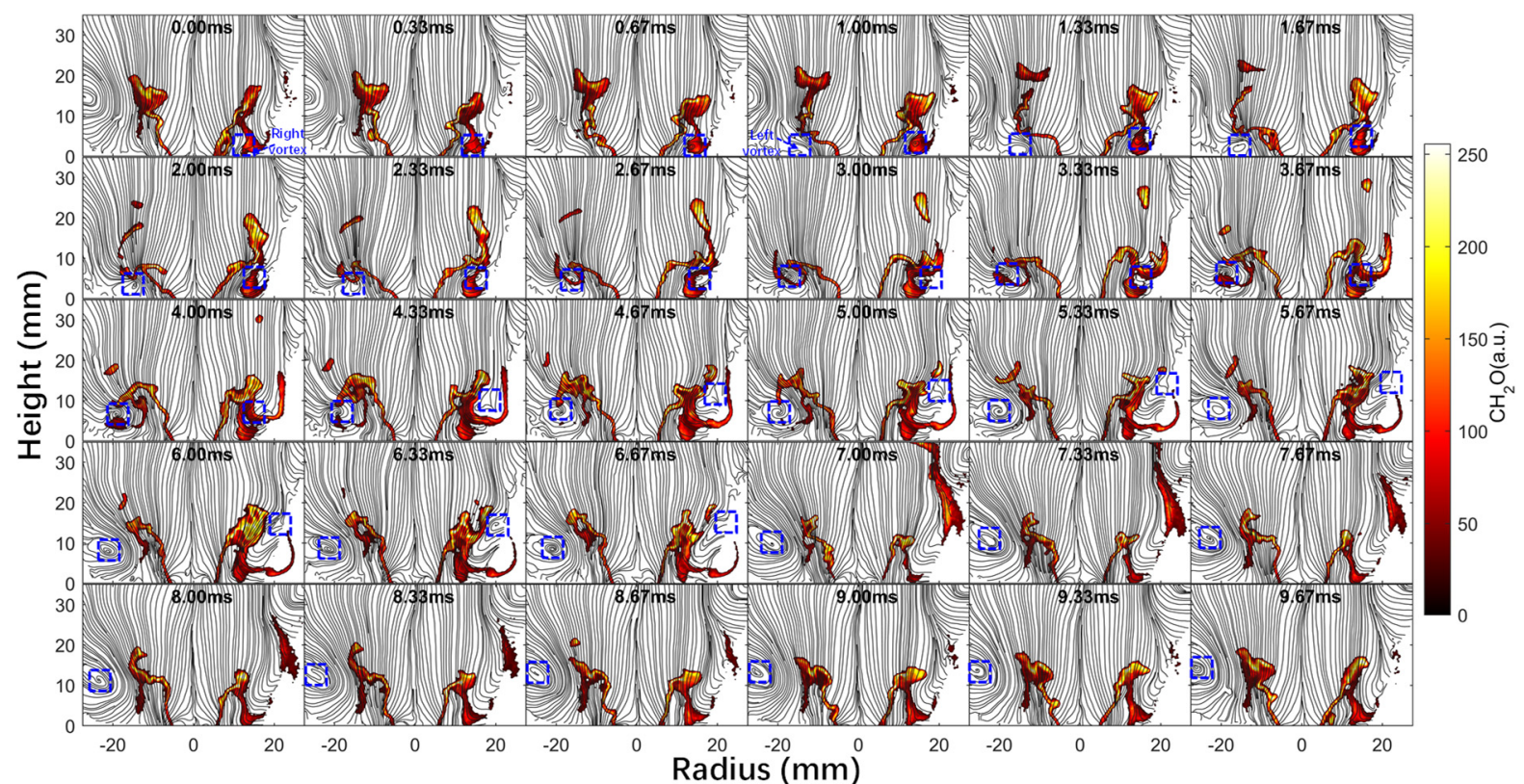

Figure 7 Time-resolved streamline and $\mathrm{CH}_{2} \mathrm{O}$ of open DME/air swirling flame at $f=100 \mathrm{~Hz}$ and $u^{\prime} / \bar{u}=0.8$.

The vortex in the flow field is very important to the present swirling flame. When the vortex is very small in 0$1.00 \mathrm{~ms}$ for the confined flame and $0-1.33 \mathrm{~ms}$ for the open flame, it has weak influence on the flame front. This is termed as the vortex generation stage. As the vortex propagates in 1.33-3.67 ms for the confined flame and 1.67$4.67 \mathrm{~ms}$ for the open flame, the vortex becomes strong and close enough to interact with the flame front. It significantly 
roll-up the flame front and changes the flame area. The flame front becomes really wrinkled and folded in this stage. When the vortex further evolves and becomes larger, its size is too large to roll-up the flame front in the confine case. However, the vortex still contribute to the flame brush by transferring peripheral air to the reaction zone in the open case. In different stages, the flame front can occasionally break up and produce small segments. These small fresh gas segments burn for several millisecond and contribute to the overall heat release fluctuation. Obviously, more detailed information, like the vortex size, position, intensity and surrounding conditions, should be studied to understand the role of acoustically induced vortex in premixed swirling flames.

In Figs. 4, 6 and 7, the base part of the flame front locates in a narrow region while the tip part disperse in a wide region. Similar phenomenon has also been studied by Palies (Palies et al., 2010) and Ranalli (Ranalli and Ferguson, 2012)using the $\mathrm{CH}^{*}$ and $\mathrm{OH}^{*}$ chemiluminescence emission images. The chemiluminescence images are divided into an upper window and a lower window. Bunce et al. (Bunce et al., 2013) systematically analyzed choice of window division and the phase synchronization. It was found that the heat release interference cannot be well explained using the rectangular window. The measured morphology of the base and tip part of the swirling flame indicate the perturbation mechanism are different. With the present averaged and time-resolved PIV and PLIF results, the mechanism of dividing the swirling flame into different parts is briefly discussed. Two main shear layers exist in the flow field. The inner shear layer is much weaker than the outer shear layer. Since the acoustically induced vortex primarily originate from the outer shear layer and moves to the peripheral zone downstream, the vortex can significantly roll-up the outer part in both open and confined cases. The vortex has so significant influence that the flame front becomes really wrinkled, folded and even break-up. The greatly enlarged flame front eventually dominate in the variation of flame front area and heat release rate. In contrast, the inner flame part can hardly perceive the vortex according to the trajectory of vortex in the outer shear layer. The inner part mainly perturbed by the periodically modulated flow velocity and random turbulence perturbation. The inner part flame front therefore shows slightly wrinkled shape and keeps relatively smooth flame front. Therefore, previous rectangular window may be feasible to the flame under small acoustic modulation amplitude, but not for the flame under very large acoustic modulation amplitude, especially for the open flame. More complicate window which distinguishes the acoustically induced vortex affected region and unaffected region can be developed.

\subsection{Dynamics of Flame Root and Angle}

According to the $\mathrm{CH}_{2} \mathrm{O}$ PLIF marked flame front in Figs. 4, 6 and 7, it can be found that both the flame root and flame base angle do not always have the same value. The flame root can move around the flow separation point of bluff body especially at large $u^{\prime} / \bar{u}$. The flame base region also becomes wider at large $u^{\prime} / \bar{u}$ which indicates the flame base sways and changes its angle dramatically. To better understand the flame root movements and the flame base angle variation, the flame root position and flame angle are extracted from the instantaneous fluorescence images as illustrated in Fig. 2. The straight region of flame front originated from the obtained flame root was recognized before significant distortion appears. Two angles between the flame base and vertical lines, namely $\theta_{\text {left }}$ and $\theta_{\text {right }}$, were extracted from the thin flame brush. The determined flame root and flame angle are used for studying the flame base dynamics. Figure 8 shows the distribution of flame root coordinates. The flame root on the right side has higher vertical coordinate than that on the left side. In the experiment, the laser sheet direction was adjusted to be parallel to the burner surface. If the flame root fluorescence signal shows a disappearing point in the laser sheet passed region, the disappearing point is considered as the root of the flame brush. As the disappearing point of the higher flame root totally locates in the laser sheet region and still has a little distance to the edge of laser sheet, the higher flame root is therefore correctly captured. The different heights of the flame roots imply that we indeed captured the flame root instead of an ambiguous point. Moreover, the left flame root shows a similar distribution of flame root coordinates. Most part of the left flame root is considered to be captured.

In Fig. 8, the flame root locates at radius larger than 5 $\mathrm{mm}$ at most time. This indicates that flame front do not always anchor at the low velocity backside of the bluff body. The radius range of the flame root position is about 1-4 mm while the vertical range is about $0.4-0.6 \mathrm{~mm}$. The flame root shows more apparent movement in the horizontal direction compared to the vertical movements. The combined random turbulent perturbation and harmonic acoustic modulation results in the movement of the flame root. As the acoustic modulation amplitude increase from 0.2 to 0.8 , the open flame root distribution only slightly varied while the confined flame root distribution becomes more disperse. The open flame root seems less sensitive to the enlarged modulation than the confined flame root.
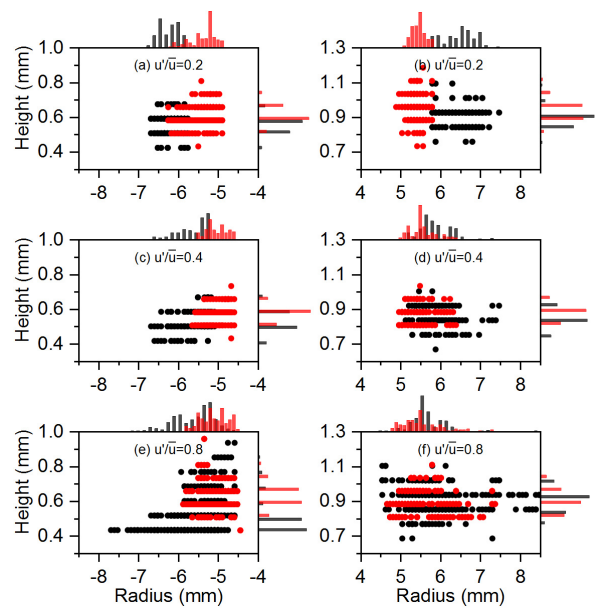

Figure $8 \mathrm{CH}_{2} \mathrm{O}$ fluorescence marked flame root distribution of DME/air swirling flame with (black) and without (red) confinement 
Besides the flame root movement, the flame angle is also very important to the heat release rate fluctuation of swirling flame (Palies et al., 2010; Candel et al., 2014). The instantaneous images in Fig. 4 have clearly shown the variation of the flame angle in one cycle of acoustic modulation. Using base region of the flame brush without significant broadening, the flame angle is determined by fitting the relatively straight range of the flame base. The left flame angle is assigned minus sign and the right flame angle is assigned plus sign. The sign of the flame angle only distinguishes the flame angle ascription. Figure 9 shows the statistic counts histogram of the flame angles. The flame angles distribute in a wide range of approximately $0-60^{\circ}$. Most time the flame angles range from $20^{\circ}$ to $40^{\circ}$ while the flame base can occasionally be almost parallel to the axial velocity. The counts bar seems to obey the normal distribution so that they are fitted using the normal distribution. It is found that the most probable flame angles are about $26^{\circ}-30^{\circ}$ according to the fitted mean values. The standard deviation of the flame angle is about $8^{\circ}-12^{\circ}$. Figure 10 plots the mean flame angle and the band of two standard deviations. In other words, $95 \%$ of the flame angles lie within two standard deviations band. The mean flame angle seems very robust. There is slight difference between the mean flame angles of the left and right flame parts. With increasing acoustic modulation amplitude, the flame angle tends to disperse in wider ranges. The open flame angles can have more clustered distribution at weak acoustic modulation but they become much dispersed under strong acoustic modulation.

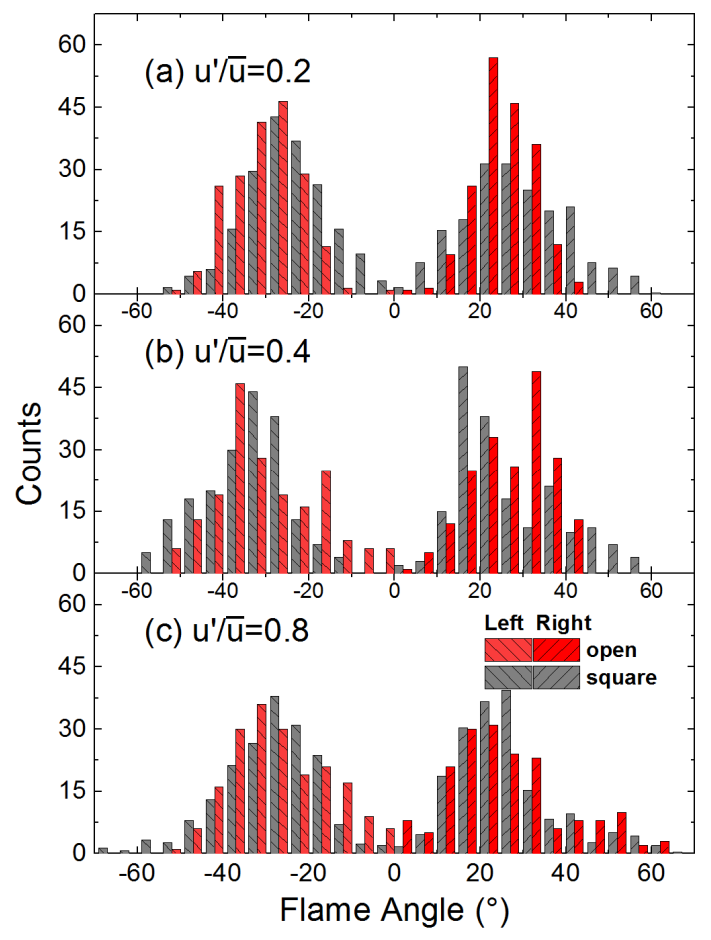

Figure 9 Extracted flame angle distribution of DME/air swirling flame with (black) and without (red) confinement.

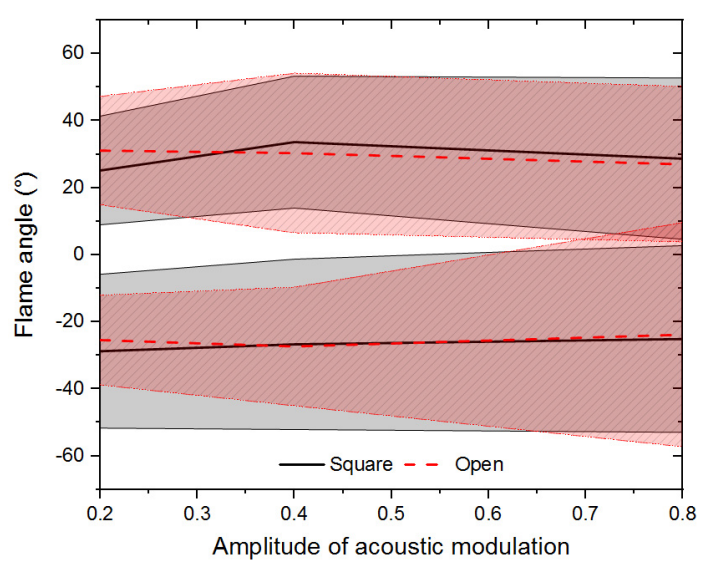

Figure 10 Mean flame angle with $95 \%$ confidence band of two standard deviations of DME/air swirling flame with (solid line) and without (dash line) confinement.

In general, the wide flame angle range indicates the flame base is greatly perturbed. The flame area and local heat release rate can be further influenced by changing the flame angle. The detailed flame base dynamics from the timeresolved results also show that the flame base deformation can be very dramatic. The deformation results in wrinkled flame base brush that can also change the local heat release rate. More instantaneous, averaged and statistic measurements are required to better understand the flame dynamics and instability of external modulated or selfexcited swirling flame.

\section{CONCLUSIONS}

A single-nozzle acoustically excited swirling burner was applied to investigate the flame dynamics of premixed stoichiometric DME/air flames with and without confinement. Simultaneous PIV and $\mathrm{CH}_{2} \mathrm{O}$ PLIF were measured using a high-speed burst-mode Nd:YAG laser with a repetition rate of $20 \mathrm{kHz}$ to obtain time-averaged and timeresolved flow field and flame front. Time-averaged flow velocity and vorticity are nearly axisymmetric. The averaged velocity vector looks similar between the flame with and without confinement. The outer shear layer is much stronger than the inner shear layer. Increasing the excitation amplitude slightly gentle the relative strength of the inner and outer shear layers. Both the left and right clouds of flame brush distributed over larger areas compared to the instantaneous flame brush. The flame brush cloud area can be enlarged by increasing acoustic amplitude and removing the confinement instead of varying acoustic frequency.

The combined streamline and flame brush depicted the continuous evolution of swirling flame. The generation, evolution, and attenuation stages of acoustically induced vortex were observed. The open case provides a larger space for the development of the vortex. The vortex significantly roll-up the flame front and changes the flame heat release in the early stage of each vortex life cycle. Since the surrounding air can mix with the combustible mixture and participate in the combustion reactions, the outer part of flame brush in the open case is much dispersed than the compact flame front in the confined case. The flame base and 
tip both suffer from periodically perturbed flow velocity and local turbulent structures. However, the origin point from the outer shear layer and the trajectory of vortex determine that the flame tip can perceive the vortex and become significantly wrinkled, folded and even break-up. The different perturbation mechanisms lead to the different flame brush cloud areas of the flame base and tip.

Flame root and flame angle are extracted from the high speed fluorescence images. Flame root shows more dramatic movement in the horizontal direction compared to the vertical movement. The open flame root seems less sensitive to the enlarged modulation than the confined flame root. The flame angle distributes in a wide range and the most probable flame angle is about $26^{\circ}-30^{\circ}$. The mean flame angle seems very robust. Open flame angles have more clustered distribution at weak acoustic modulation while they become very dispersed under strong acoustic modulation.

According to the present observation, the confinement mainly affects the outer part flame through changing the coherent vortex structure. The flame dynamics and heat release fluctuation are thus affected which could change the thermoacoustic instability of a combustion system.

\section{ACKNOWLEDGMENTS}

This research is supported by National Natural Science Foundation of China (91541201, 51676126, 51606123) and Shanghai Science and Technology Committee (no. 17XD1402000).

\section{REFERENCES}

Allison P. M., Chen Y., Ihme M. and Driscoll J. F. (2015). Coupling of flame geometry and combustion instabilities based on kilohertz formaldehyde PLIF measurements. Proceedings of the Combustion Institute, (35), 3255-3262.

Bellows B. D., Bobba M. K., Forte A., Seitzman J. M. and Lieuwen T. (2007). Flame transfer function saturation mechanisms in a swirl-stabilized combustor. Proceedings of the Combustion Institute, (31), 3181-3188.

Bellows B. D., Bobba M. K., Seitzman J. M. and Lieuwen T. (2006). Nonlinear flame transfer function characteristics in a swirl-stabilized combustor. Journal of Engineering for Gas Turbines and Power, (129), 954-961.

Birbaud A. L., Durox D., Ducruix S. and Candel S. (2007). Dynamics of confined premixed flames submitted to upstream acoustic modulations. Proceedings of the Combustion Institute, (31), 1257-1265.

Boxx I., Stöhr M., Carter C. and Meier W. (2010). Temporally resolved planar measurements of transient phenomena in a partially pre-mixed swirl flame in a gas turbine model combustor. Combustion and Flame, (157), 1510-1525.

Bunce N. A., Quay B. D. and Santavicca D. A. (2013). Interaction between swirl number fluctuations and vortex shedding in a single-nozzle turbulent swirling fully-premixed combustor. Journal of Engineering for Gas Turbines and Power, (136), 021503-021503-11.

Candel S., Durox D., Schuller T., Bourgouin J.-F. and Moeck J. P. (2014). Dynamics of swirling flames. Annual Review of Fluid Mechanics, (46), 147-173.
Cuquel A., Durox D. and Schuller T. (2013). Scaling the flame transfer function of confined premixed conical flames. Proceedings of the Combustion Institute, (34), 1007-1014.

De Rosa A. J., Peluso S. J., Quay B. D. and Santavicca D. A. (2015). The effect of confinement on the structure and dynamic response of lean-premixed, swirl-stabilized flames. Journal of Engineering for Gas Turbines and Power, (138), 061507.

De Rosa A. J., Peluso S. J., Quay B. D. and Santavicca D. A. (2017). Lean-premixed, swirl-stabilized flame response: flame structure and response as a function of confinement. Journal of Engineering for Gas Turbines and Power, (140), 031504

Fu Y., Cai J., Jeng S.-M. and Mongia H. Confinement effects on the swirling flow of a counter-rotating swirl cup. ASME Turbo Expo 2005: Power for Land, Sea, and Air, 2005 Reno, Nevada, USA. GT2005-68622, pp469-478.

Fugger C. A., Roy S., Caswell A. W., Rankin B. A. and Gord J. R. (2018). Structure and dynamics of $\mathrm{CH}_{2} \mathrm{O}, \mathrm{OH}$, and the velocity field of a confined bluff-body premixed flame, using simultaneous PLIF and PIV at $10 \mathrm{kHz}$. Proceedings of the Combustion Institute, (37), 1461-1469.

Huang Y. and Yang V. (2009). Dynamics and stability of lean-premixed swirl-stabilized combustion. Progress in Energy and Combustion Science, (35), 293-364.

Lieuwen T. C. (2014). Unsteady Combustor Physics, Cambridge University Press.

Meier W., Weigand P., Duan X. R. and GiezendannerThoben R. (2007). Detailed characterization of the dynamics of thermoacoustic pulsations in a lean premixed swirl flame. Combustion and Flame, (150), 2-26.

Palies P., Durox D., Schuller T. and Candel S. (2010). The combined dynamics of swirler and turbulent premixed swirling flames. Combustion and Flame, (157), 1698-1717.

Poinsot T. (2017). Prediction and control of combustion instabilities in real engines. Proceedings of the Combustion Institute, (36), 1-28.

Ranalli J. and Ferguson D. (2012). Measurement of flame frequency response functions under exhaust gas recirculation conditions. Journal of Engineering for Gas Turbines and Power, (134), 091502-091502-10.

Stöhr M., Boxx I., Carter C. D. and Meier W. (2012). Experimental study of vortex-flame interaction in a gas turbine model combustor. Combustion and Flame, (159), 2636-2649.

Wabel T. M., Skiba A. W., Temme J. E. and Driscoll J. F. (2017). Measurements to determine the regimes of premixed flames in extreme turbulence. Proceedings of the Combustion Institute, (36), 1809-1816.

Wang G., Liu X., Wang S., Li L. and Qi F. (2019). Experimental investigation of entropy waves generated from acoustically excited premixed swirling flame. Combustion and Flame, (204), 85-102.

Weigand P., Meier W., Duan X. R., Stricker W. and Aigner M. (2006). Investigations of swirl flames in a gas turbine model combustor: I. Flow field, structures, temperature, and species distributions. Combustion and Flame, (144), 205224. 